\title{
NUEVO REGIONALISMO INTERNACIONAL: SUS EXPRESIONES EN LATINOAMÉRICA.
}

Autor: John Arturo Buelvas Parra (1)

\section{RESUMEN}

Debido al progresivo crecimiento de la globalización y su influencia en muchos países que aprovechando sus ubicaciones geográficas para la comercialización de sus productos ha permitido por la proximidad de sus territorios al proceso de integración regional a través de acuerdos entre ellos con la finalidad de reducir barreras comerciales que limitan la libre circulación de bienes, servicios y factores de producción entre los países firmantes del acuerdo, para lo que optaron por medidas que como la reducción de los impedimentos arancelarios y no arancelarios logrando de esta manera un mejor aprovechamiento de los flujos comerciales entre los países que hacen parte de la integración regional. la integración en Latinoamérica no ha tenido la satisfacción esperada a causa de las discordias, que en algunos casos ha provocado el rompimiento de relaciones entre los países participantes, dado que el país más fuerte busca imponer su política económica. Son distintos los factores que pueden influir en el éxito en los procesos de integración entre los diversos países, como lo es la certeza de cierto grado de armonización de las políticas económicas y comerciales, las cuales deben incluir la monetaria y fiscal, a su vez las de circulación de factores productivos, sin desconocer que este tipo de acuerdos comerciales debe plantear hasta qué punto resulta conveniente para cada país integrante.

Palabras claves: Globalización, integración regional, acuerdos de cooperación

\section{ABSTRACT}

Due to the progressive growth of globalization and its influence in many countries, the use of its geographical locations for the commercialization of its products has allowed the proximity of its territories to the process of regional integration through agreements among them to reduce trade barriers. that limit the free circulation of goods, services and factors of production among the countries that signed the agreement, for which they opted for measures such as the reduction of tariff and non-tariff impediments, thus achieving a better use of trade flows between countries that They are part of regional integration. Integration in Latin America has not had the expected satisfaction due to the discord, which in some cases has caused the breakdown of relations between the participating countries, given that the strongest country seeks to impose its economic policy. There are different factors that can influence the success of integration processes between different countries, such as the certainty of a certain degree of harmonization of economic and trade policies, which must include monetary and fiscal policies, in turn, the circulation of factors productive, without ignoring that this type of commercial agreements must consider to what extent it is convenient for each member country

Keywords: Globalization, regional integration, cooperation agreements 
NUEVO REGIONALISMO INTERNACIONAL: SUS EXPRESIONES EN LATINOAMÉRICA

\section{INTRODUCCIÓN}

7 s importante destacar que los procesos de inte$\triangle$ gración cobraron protagonismo e importancia de significancia económica una vez finalizó la segunda guerra mundial, y esta unificación se encontraba fundamentada en las motivaciones políticas y económicas.

Pese a la progresiva tendencia de globalización, la cual ha encaminado a los países ubicados geográficamente con proximidad de sus territorios al proceso de integración regional a través de acuerdos entre ellos con la finalidad de reducir barreras comerciales que limitan la libre circulación de bienes, servicios y factores de producción entre los países firmantes del acuerdo, para lo que optaron por medidas que como la reducción de los impedimentos arancelarios y no arancelarios logrando de esta manera un mejor aprovechamiento de los flujos comerciales entre los países que hacen parte de la integración regional. Sin embargo en algunos casos la creación de bloques económicos ha permitido el fomento de costos elevados dado que han establecido en sus políticas económicas a un solo país como a proveedor de la producción dentro del bloque originando efectos negativos a los países miembros y por ende a la población social.

El interés de los países al conformar bloques económicos radica principalmente en constituir un área de libre comercio, lo que les dará como ventajas una mayor posibilidad en el desarrollo económico y tecnológico entre los países, así mismo mejores posibilidades para ser competentes con las demás regiones del mundo y de esta manera formar un frente común para enfrentar las amenazas externas.

Cabe resaltar que las integraciones regionales son un medio para alcanzar un mayor desarrollo económico en aras de satisfacer las necesidades de toda la población social que hacen parte de dicha integración, contribuyendo así al bienestar general.

\section{Nuevo Regionalismo Internacional: sus expresio- nes en Latinoamérica.}

Conceptualización sobre Regionalismo: Según lo planteado por el autor del artículo, el regionalismo se sustenta en la idea de región, es decir, en un limitado número de Estados ligados geográficamente y con un alto grado de interdependencia entre ellos. De igual forma la CEPAL (Sardo, 2011), habla de un Regionalismo Abierto, y que es usado en América Latina y los define como "un proceso que busca conciliar la interdependencia nacida de acuerdos comerciales preferenciales y la interdependencia impulsada por las señales del mercado resultante de la liberación comercial en general, donde las políticas explicitas de integración sean compatibles con las políticas tendientes a elevar la competitividad internacional y que las complementen".

Para expresar este concepto el autor plantea que existen tres corrientes cuyas variables explicativas son diferentes. El primero se refiere a la teoría sistémica (neorrealismo y globalización), se basa en la importancia de las estructuras políticas y económicas ampliadas y el impacto de las presiones externas sobre la región. El segundo se refiere a las teorías de la interdependencia (neofuncionalismo, institucionalismo neoliberal y constructivismo), el cual parte de motivos de interdependencia creciente entre los actores involucrados. Como tercero se tiene a las teorías domésticas (coherencia estatal, tipo de régimen y democratización y teorías de convergencia), parten de los principios de comunidad en etnicidad, raza, idioma, religión, cultura, historia y conciencia de una herencia común. (Ver Gráfico 1). 
NUEVO REGIONALISMO INTERNACIONAL: SUS EXPRESIONES EN LATINOAMÉRICA

Gráfico 1. Teorías Sobre Regionalismo.

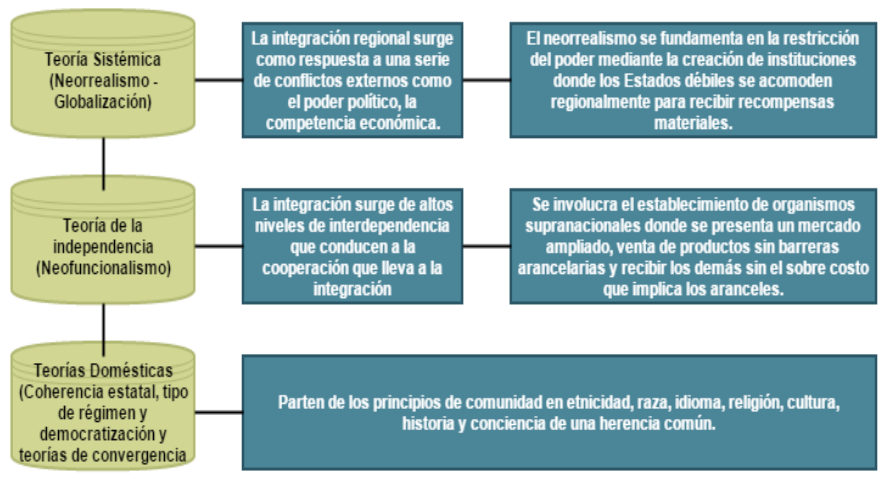

Fuente: Elaborada por el autor.

En ese mismo orden de ideas, el concepto de integración regional aborda el concepto de integración económica, y la cual también presenta tres posturas ideológicas distintas como son: la liberal, la estructural y la dirigista.

La orientación liberal se inclina realmente a la integración mundial, la estructural se orienta hacia el mercado y a la parte de rentabilidad que se puede generar en el intercambio mercantil, y la dirigista está realmente al mando del estado como determinante de todas aquellas políticas de tipo mercantiles y de intercambio. (Ver gráfico 2.)

Gráfico 2. Posturas o enfoques de integración económica.

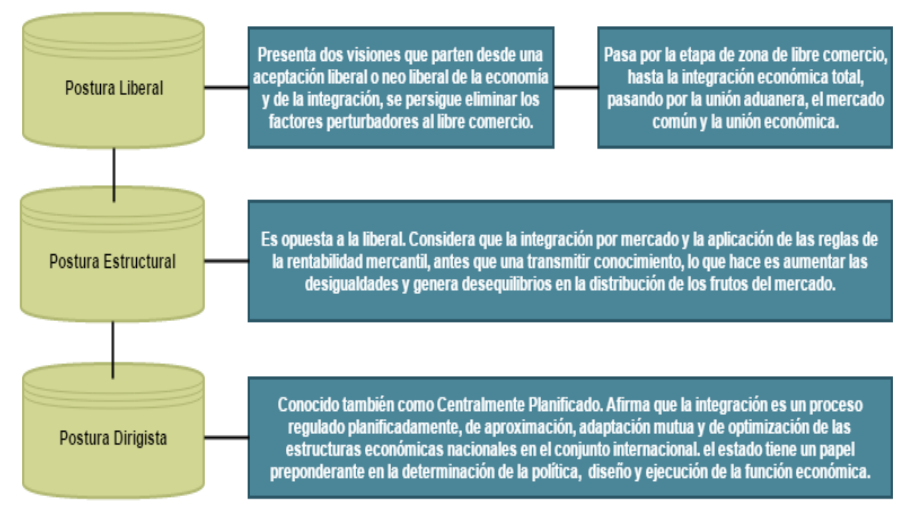

Fuente: Elaborada por el autor.

\section{Motivadores que dan origen al Nuevo Regiona- lismo:}

Hechos históricos: el denominado proceso de globalización de la economía mundial, de igual forma los cambios políticos y económicos generados en el denominado tercer mundo. De igual forma lo sucedido al finalizar la guerra fría dando origen a nuevos sistemas políticos y económicos de gran ambición, en un mundo de mayor democratización y liberación. Otro aspecto a resaltar es el dominio de grandes potencias como EE. UU, quien en unión con la Unión europea han impuesto nuevas políticas de comercio en el ámbito internacional.

La crisis financiera asiática: (Hernandez Mangones, 2006), en la década de los 90 el continente asiático presenta su mayor crisis, por la quiebra de instituciones financieras japonesas, empresas como Maruso Securities, Ogawa Securities, Sanyo entre otras, quedaron en la quiebra por la caída de las cotizaciones en la bolsa de Tokio y a operaciones no rentables efectuadas y debido a la salida de la inversión extranjera, Asia se fue quedando sin dinero del mundo y por el dominio de la economía norte americana en esos años.

La intensificación de las relaciones de cooperación del siglo XX, originadas después de la segunda guerra mundial.

Tres etapas han contribuido con el proceso de regionalización en américa latina. La primera comprendida desde lo finales de los años 50 y principios de los 60, cuando surge la ALALC (Asociación Latinoamericana de Libre Comercio) y más tarde el MCCA (Mercado Común Centroamericano). La segunda se presenta al finalizar la década de los 60, cuando se crea el GA (Grupo Andino) y el CAPRICOM (Mercado Común del Caribe). Y como tercera etapa, en la década de los 90, se conforma el MERCOSUR (Mercado Común Sudamericano) y el denominado TLCAN (Tratado de Libre Comercio de América del Norte). 


\section{NUEVO REGIONALISMO INTERNACIONAL: SUS EXPRESIONES EN LATINOAMÉRICA}

EI Nuevo Regionalismo y sus consecuencias en Latinoamérica:

El único proceso que ha contribuido como nexo entre el denominado viejo regionalismo y el nuevo regionalismo es el CAN (Comunidad Andina de naciones). En este sentido para que el nuevo regionalismo se diera con fuerza, era necesario que el viejo regionalismo cediera su puesto. Esto porque el viejo regionalismo tuvo grandes obstáculos como el generado por el proteccionismo de los estados a la apertura de nuevos mercados regionales y que eran sometidos por los gobiernos autoritarios.

Es así como surge el nuevo Regionalismo abierto, producto de la integración latinoamericana y en él se combina la liberación interna y externa con el fin de mejorar la eficiencia productiva y acrecentar una sólida competitividad en los mercados regionales e internacionales.

Pero esta integración también presenta obstáculos notorios como: la diferencias en la repartición de beneficios entre los países integrantes, el alto costo económico que requiere mantener la estructura institucional creada, la falta de capacidad para construir acuerdos ajustados a las necesidades de cada país.

\section{UBICACIÓN DE COLOMBIA EN LOS PRO- CESOS DE INTEGRACIÓN REGIONAL.}

Colombia, es un país que se encuentra a la vanguardia en Latinoamérica en procesos de integración. Es así como lo reporta el Gobierno de (España., 2013), quien afirma que "los procesos de integración en América latina viven un renovado impulso político $\mathrm{y}$, pese a los obstáculos, avanzan en diferentes frentes y a diferentes velocidades. Actualmente hay vigentes múltiples organizaciones regionales o subregionales de integración y concertación. El proceso de integración más reciente es la Alianza del Pacifico. Entro los organismos de integración cabe desta- car MERCOSUR, CAN, CARICOM, UNASUR, SICA, ALBA, la Alianza del Pacífico y CELAC". El Ministerio de Relaciones (Exteriores, 2015) de Colombia, en su pagina http:// www.cancilleria.gov.co/, reporta que Colombia, es un país inmerso en grandes procesos de Integración Regional y Subregionales (Ver Cuadro 1).

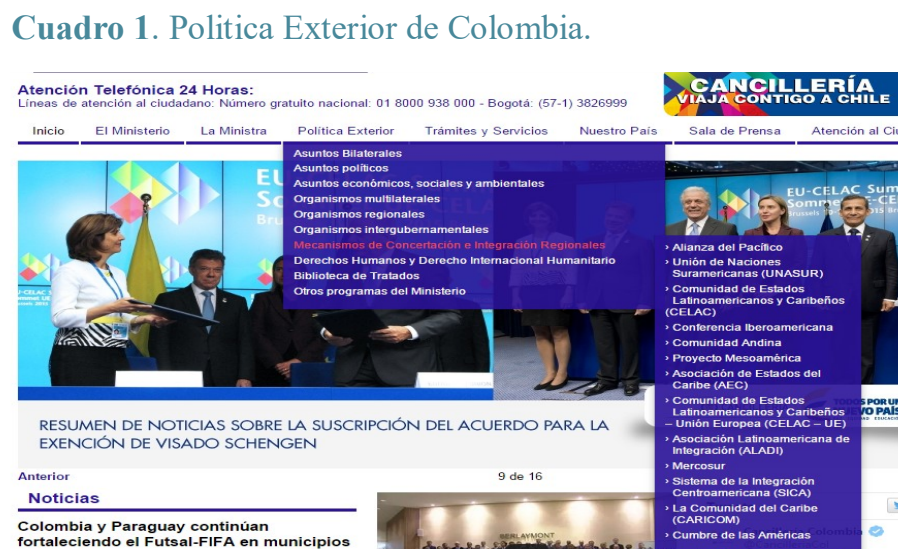

Fuente: http://www.cancilleria.gov.co/.

Como se observa en el cuadro 1. Los mecanismos de concertación e integración regionales a los que hace parte Colombia se resumen de la siguiente manera en los gráficos siguientes: Alianza del pacífico, Unión de Naciones Suramericanas (UNASUR), Comunidad de Estados latinoamericanos y Caribeños

Gráfico 3. Mecanismos de Integración Regional de Colombia.

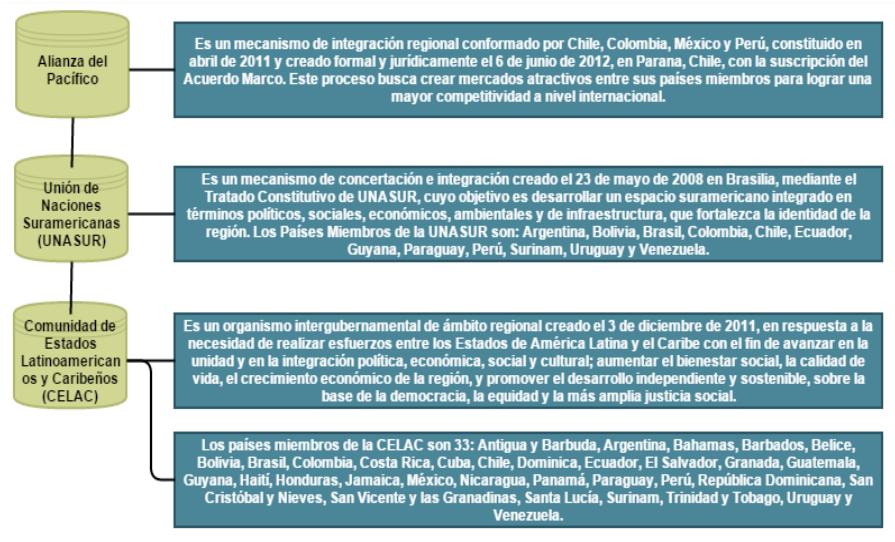

Fuente: Cancillería Colombiana. Adaptado por el autor. 


\section{NUEVO REGIONALISMO INTERNACIONAL: SUS EXPRESIONES EN LATINOAMÉRICA}

La Conferencia iberoamericana, la comunidad Andina (CA), el Proyecto Mesoamérica.

Gráfico 4. Mecanismos de Integración Regional de Colombia.

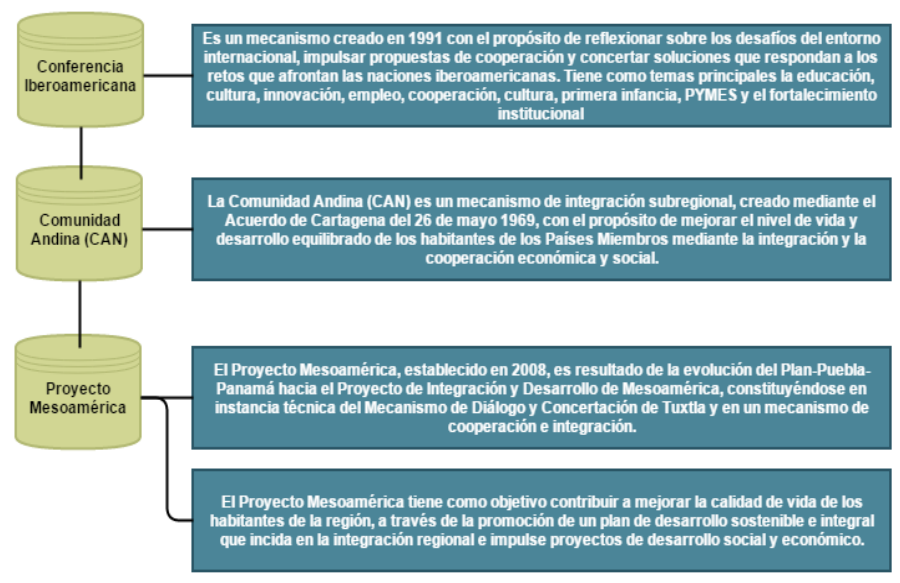

Fuente: Cancillería Colombiana. Adaptado por el autor.

Otros de los mecanismos de integración en los cuales participa Colombia, son: la Asociación de Estados del Caribe, la comunidad de Estados latinoamericanos y Caribeños en conjunto con la Unión Europea. La Asociación latinoamericana de integración (ALADI).

Gráfico 5. Mecanismos de Integración Regional de Colombia.

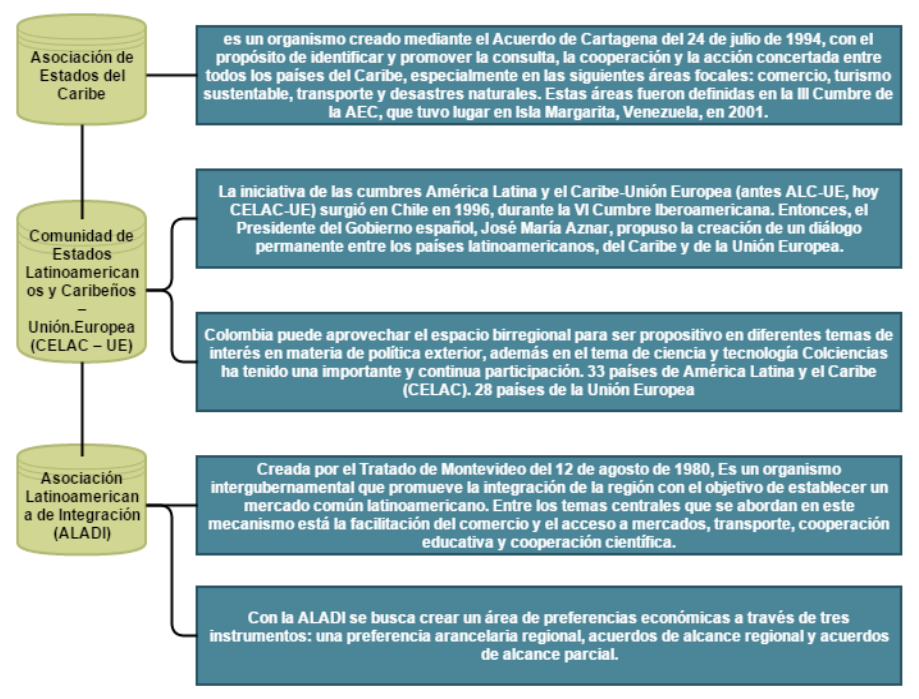

Fuente: Cancillería Colombiana. Adaptado por el autor.
Hace parte también del Mercosur, el Sistema de la integración Centroamericana (SICA), de la Comunidad del Caribe (CARICOM), y de la Cumbre de las Américas.

Gráfico 5. Mecanismos de Integración Regional de Colombia.

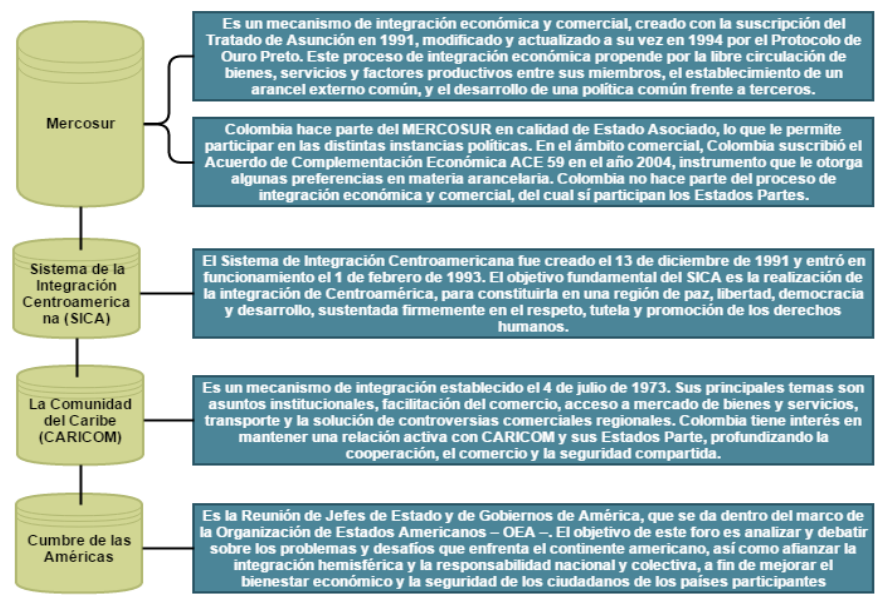

Fuente: Cancillería Colombiana. Adaptado por el autor

\section{VENTAJAS Y DESVENTAJAS DE LOS PRO- CESOS DE INTEGRACIÓN REGIONAL CO- MERCIAL.}

Es bueno destacar que dentro de las ventajas más importantes que se puede presentar en un proceso de integración regional comercial es lo relacionado con el avance en los procesos de industrialización y obtención de las llamadas economías de escalas. Así mismo se mejorar las relaciones entre los países que lo conforman y se incrementa la capacidad de negociación entre los países. 
NUEVO REGIONALISMO INTERNACIONAL: SUS EXPRESIONES EN LATINOAMÉRICA

Otro punto importante a destacar es el impulso que se da a la tendencia de unificación de espacios geopolíticos, bloques de países facilitando así las relaciones entre sus integrantes. Así mismo en estos procesos se fortalecen los procesos democráticos por la participación libre que se puede generar. (Ver Gráfico 3).

Gráfico 3. Proceso de Integración Regional.

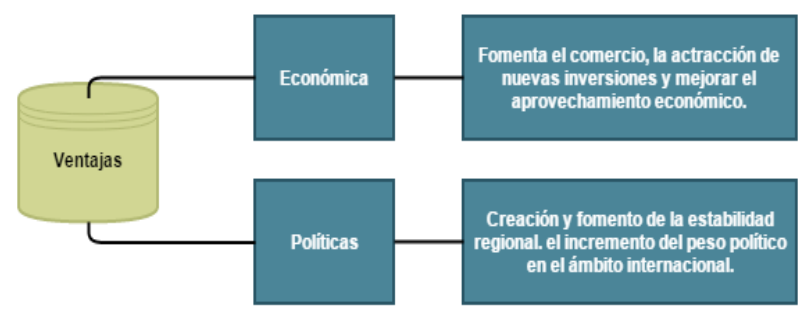

Fuente: Elaborado por el autor.

En este proceso de análisis, nos encontramos con las grandes desventajas o riesgos que se pueden presentar en este tipo de proceso. Es el caso del posible decaimiento del comercio producto de los incumplimientos de compromisos pactados en dichos acuerdos. También se presenta falta de equidad en la distribución de costos y beneficios. Además de la debilidad en la eficiencia económica y operativa, también cabe resaltar que en muchos casos se presenta incompatibilidad de objetivos y políticas económicas como el caso del rompimiento con algún esquema de integración en el caso de la salida de Chile del Grupo Andino.

\section{Gráfico 4. Proceso de integración Regional}

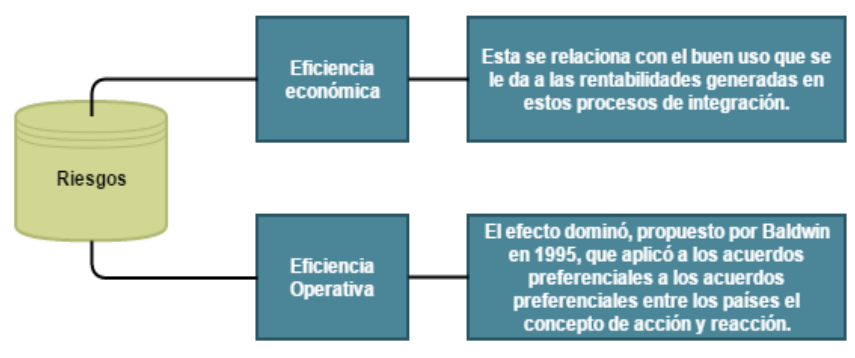

Fuente: Elaborado por el autor.

\section{CONCLUSIONES.}

Todo proceso de integración regional debería establecer previamente las políticas a seguir por todos los países que hacen parte de ella. Partiendo desde el libre comercio, hasta llegar la unificación de la económica y la moneda monetaria, para que finalmente se dé la unión gubernamental, por lo que necesariamente esta situación implicaría la constitución de organismos con la facultad de dictar normas de cumplimiento obligatorio e inmediato para los Estados.

Por otra parte, la integración en Latinoamérica no ha tenido la satisfacción esperada a causa de las discordias, que en algunos casos ha provocado el rompimiento de relaciones entre los países participantes, dado que el país más fuerte busca imponer su política económica.

No obstante la organización en bloques regionales representa potenciales ventajas como el fomento del comercio, mayor inversión en los países que se asocian a la integración regional y un mayor aprovechamiento de sus economías y en general mejores niveles de bienestar entre las naciones, sin embargo frente al hecho evidente de que un proceso de integración, ya sea en sus aspectos políticos como económicos, es más difícil en presencia de factores como las desigualdades económicas tales como el subdesarrollo, lo que finalmente repercute en la inestabilidad y el descontento social de los pueblos. A pesar de ello estos procesos de integración han permitido un mayor dinamismo en los intercambios comerciales, lo cual los ha facultado en la inserción internacional de las economías intervinientes.

Se puede asumir que son distintos los factores que pueden influir en el éxito en los procesos de integración entre los diversos países, como lo es la certeza de cierto grado de armonización de las políticas económicas y comerciales, las cuales deben incluir la monetaria y fiscal, a su vez las de circula- 


\section{NUEVO REGIONALISMO INTERNACIONAL: SUS EXPRESIONES EN LATINOAMÉRICA}

ción de factores productivos, sin desconocer que este tipo de acuerdos comerciales debe plantear hasta qué punto resulta conveniente para cada país integrante.

\section{BIBLIOGRAFIA.}

España., G. d. (2013). Procesos de integración regional. Madrid. España.: Cancilleria.

Exteriores, M. d. (02 de 06 de 2015). http:// www.cancilleria.gov.co/. Recuperado el 19 de 06 de 2015.

Hernandez Mangones, G. (2006). Diccionario de Economía. Bogotá:: Universidad Cooperativa de Colombia.

Sardo, D. E. (2011). La Triple Frontera entre Argentina, Brasil y paraguay. ¿Una macro región basada en el concepto de regionalismo abierto?. New York. United States.: Lulu Press international. 